In Cres. Vol. $3 N^{\circ}$ 2: pp. 267-276, 2012

\title{
ESTILO DE VIDA Y ESTADO NUTRICIONAL DEL ADULTO MAYOR*
}

\author{
LIFE STYLE AND NUTRITIONAL STATUS \\ OF THE ELDERLY
}

Catherine Torrejón Mendoza $a^{1}$ Elena Reyna Márquez²

\section{RESUMEN}

El presente trabajo de investigación es de tipo cuantitativo, con diseño descriptivo correlacional. El objetivo fue determinar la relación que existe entre estilo de vida y estado nutricional del adulto mayor de la H.U.P Javier Heraud - Santa. La muestra estuvo constituida por 143 adultos mayores. Para la recolección de datos se utilizaron dos instrumentos: la escala de estilo de vida y la tabla de valoración nutricional según índice de masa corporal para el adulto mayor de 60 años a más. El análisis y procesamiento de datos se realizó en el Software SPSS versión 18.0. Se aplicó la prueba estadística de independencia de criterios Chi Cuadrado, con $95 \%$ de confianza, para determinar la relación que existe entre las variables, llegándose a las siguientes conclusiones: Más de la mitad de los adultos mayores presentan un estilo de vida no saludable y un menor porcentaje, saludable. En relación al estado nutricional se encontró que menos de la mitad de los adultos mayores presentan estado nutricional normal; más de la cuarta parte, sobrepeso; menos de la quinta parte, delgadez; y más de la décima parte, obesidad. No existe relación estadísticamente significativa entre el estilo de vida y el estado nutricional en el adulto mayor de la H. U. P. "Javier Heraud" del distrito de Santa.

PALABRAS ClAVE: Estilo vida, Estado nutricional, Adulto mayor.

* Recibido: 04 de mayo del 2012; aprobado: 25 de octubre del 2012.

1 Bachiller Escuela Profesional de Enfermería Universidad Católica Los Ángeles de Chimbote.

2 Docente de la Escuela Profesional de Enfermería Universidad Católica Los Ángeles de Chimbote. 


\section{ABSTRACT}

This nonexperimental research of descriptive corelational design aimed to establish the relationship between life style and nutritional status of the elderly at Javier Eraud shanty town-Santa. The sample consisted of 143 elderly. Two were the data collection instruments used; The lifestyle scale and the Nutritional table according to the body mass index of aged 60 and above. The SPSS version 18.0 was used for dataprocessing. Results were statistically analyzed by means of the Chi Square test for independence. The study came to the following conclusions: More than half of the elderly present unhealthy lifestyle while a minority present healthy one. Respect to the nutritional status less than half of the elderly present an average nutritional status, more than one-fourth are overweight, less than one-fifth are thin and more than one-tenth are obese. There is no statistically significant relationship between lifestyle and nutritional status of the elderly at Javier Eraud shanty town-Santa.

KEY WORDS: Lifestyle, Nutritional status, Elderly.

\section{INTRODUCCIÓN}

La preservación de la salud física y mental constituye una responsabilidad individual, con independencia de las acciones dirigidas a su conservación en el ciudadano y representa un deber cívico para evitar añadir una carga económica o sentimental a corto o lejano plazo, a la familia o a la propia sociedad, dando la posibilidad de alcanzar una vejez sana, portadora de ricas y ejemplarizantes experiencias para los más jóvenes; tal legado es un estilo de vida. ${ }^{1}$

En el país han determinado un aumento de la expectativa de vida y un envejecimiento progresivo de la población. Los adultos mayores representan más del $10 \%$ de los habitantes, grupo que presenta una alta prevalencia de hipertensión, sobrepeso, osteoporosis, problemas articulares, cáncer y trastornos cardiovasculares. $^{2}$

La población peruana para el 2007, según el Instituto Nacional de Estadística e Informática (INEI), es de 28220764 habitantes, estimándose en 2495 866 los adultos mayores, que representan el $9,1 \%$ de la población nacional, siendo la población adulta mayor varón de 1205259 (48,3\%) y adulta mayor mujer de 1290607 (51,7\%). Según el INEI, desde las últimas décadas se observa un incremento paulatino y persistente de la población adulta mayor, lo cual genera nuevas demandas en el campo de la salud y seguridad social. ${ }^{3,4}$

Aunque tradicionalmente se atribuyen los problemas de salud en el adulto mayor al proceso de envejecimiento, no sólo se evidencian decrementos, sino también incrementos funcionales; pero se reconocen cada vez más los problemas de salud en el adulto mayor, como dependientes de las relaciones entre el 
comportamiento del individuo y su contexto. Hay entonces, modos de vivir y de envejecer saludables y estilos de vida poco saludables que conducen al deterioro. ${ }^{5}$

Uno de los aspectos esenciales a tener en cuenta durante el envejecimiento es el estado nutricional del adulto mayor, que se ve afectado por los malos hábitos sanitarios mantenidos durante toda la vida, además de los trastornos que surgen del proceso de envejecimiento. ${ }^{6}$

Arancibia, R. y Hernández, D. ${ }^{7}$, en su estudio descriptivo Estado nutricional y actividad física en el adulto mayor en una población de referencia de la Provincia Ciego de Ávila, Cuba, en el año 2008, en sus resultados obtuvieron que en el Grupo I masculino las condiciones de obesidad $(12,21 \%)$, sobrepeso $(33,72 \%)$ y bajo peso $(5,81 \%)$ exceden en su conjunto los valores del normo peso expresados en este grupo (48,26\%). Cid P, Merino J, Stiepovich J. ${ }^{8}$, en Chile, en el 2006, estudiaron los Factores biológicos y psicosociales predictores del estilo de vida promotor de salud, concluyendo que el 52,5\% de la muestra tiene un estilo de vida saludable.

A nivel nacional, el Ministerio de Salud reportó que la prevalencia de desnutrición en adultos mayores es más alta que la encontrada en menores de 60 años; mencionan que más de la mitad de las personas mayores de 60 años que se controlan en consultorios del sistema público de salud, tienen problemas de sobrepeso u obesidad. ${ }^{9}$

A esta realidad problemática no escapa el adulto mayor de la H. U. P. "Javier Heraud", que se encuentra dentro de la jurisdicción del "Centro de Salud Santa". Los adultos mayores que habitan este lugar son de bajos recursos económicos, constituido principalmente por agricultores en un $90 \%$, y se dedican a la siembra y cosecha de cultivos como: maíz, papa, panca, camote, fríjol, hortalizas, arroz, etc. ${ }^{10}$

\section{PROBLEMA}

¿Existe relación entre el estilo de vida y el estado nutricional del Adulto Mayor de la H. U. P. “Javier Heraud”- Santa, 2011?

\section{OBJETIVO GENERAL}

Determinar la relación entre el estilo de vida y el estado nutricional del adulto mayor de la H.U.P Javier Heraud-Santa. 


\section{OBJETIVOS ESPECÍFICOS}

1. Valorar el estilo de vida del adulto mayor de la H.U.P "Javier Heraud" Santa.

2. Identificar el estado nutricional del adulto mayor de la H.U.P "Javier Heraud" - Santa.

El presente trabajo de investigación tiene como bases teóricas al estilo de vida, estado nutricional y características del adulto mayor.

En la etapa adulta, todo ser humano puede optar por diferentes estilos de vida; esto influirá claramente en el desarrollo y bienestar del organismo, ya que mientras más saludable y mayores cuidados tengan, mejor calidad de vida se obtendrá; así como también mejores condiciones para enfrentar el periodo de la vida que le sigue: la vejez. ${ }^{11}$

Los estilos de vida de un grupo social comprenden una serie de pautas determinadas socialmente y de interpretaciones de situaciones sociales. Estas pautas son desarrolladas y utilizadas por el grupo como mecanismos para afrontar los problemas de la vida. El estilo de vida de una persona está compuesto por sus reacciones habituales y por las pautas de conducta que haya desarrollado durante los procesos de socialización. ${ }^{12}$

Entonces, desde una perspectiva integral, los estilos de vida no pueden ser aislados del contexto social, económico, político y cultural al cual pertenecen, y deben ser acordes a los objetivos del proceso de desarrollo, que es dignificar a la persona humana en la sociedad, a través de mejores condiciones de vida y de bienestar. ${ }^{13}$

Los comportamientos o conductas son determinantes decisivos de la salud física y mental y del estado de la salud pública, estando fuertemente vinculados al bienestar. Se calcula que un tercio de las enfermedades en el mundo pueden prevenirse mediante cambios en el comportamiento. ${ }^{14}$

Las dimensiones del estilo de vida, son las siguientes:

Dimensión de Alimentación: es el mecanismo mediante el cual el organismo se alimenta de una dieta equilibrada compuesta de proteínas, carbohidratos, grasas, vitaminas, sales minerales y agua, en cantidades suficientes para conservar las funciones del adulto mayor. ${ }^{15}$

Dimensión Actividad y Ejercicio: es el esfuerzo corporal o actividad física que pone en movimiento al cuerpo en su totalidad o una parte del mismo de manera armónica, ordenada y repetida, que se realiza regularmente con el propósito de mejorar, desarrollar y conservar las funciones del adulto mayor. ${ }^{16}$ 
Dimensión Manejo del Estrés: el manejo del estrés se refiere al esfuerzo que hace el adulto mayor por controlar y reducir la tensión que surge cuando la situación es considerada difícil o inmanejable, mediante acciones coordinadas, sencillas o complejas, para sobrellevar la presión, las emergencias o el estrés. ${ }^{17}$

Dimensión Apoyo Interpersonal: es una dimensión importante por considerar al adulto mayor como ser eminentemente social, para lo cual él requiere de redes de apoyo familiar y social, lo mismo que le permita sostenerse y seguir creciendo en el camino, junto a otros individuos de su entorno. ${ }^{18}$

Dimensión Autorrealización: se refiere a la adopción del adulto mayor a su inicio como persona en la tercera edad, teniendo como tendencia innata a desarrollar al máximo los propios talentos y potenciales, que contribuyen a obtener un sentimiento de satisfacción hacia él mismo como adulto mayor, alcanzando la superación y a la autorrealización. ${ }^{19}$

Dimensión Responsabilidad en Salud: en el adulto mayor implica saber escoger y decidir sobre alternativas saludables y correctas, en vez de aquellas que generan un riesgo para su salud; además, modificar su comportamiento y realizar actividades que mantengan su salud. ${ }^{20}$

\section{METODOLOGÍA}

El presente trabajo de investigación es de tipo cuantitativo, con diseño descriptivo correlacional. La muestra está constituida por 143 adultos mayores. Para la recolección de datos, se utilizaron dos instrumentos: la escala de estilo de vida y la tabla de valoración nutricional según índice de masa corporal para el adulto mayor de 60 años a más.

Se informó y pidió consentimiento de los adultos mayores del H.U.P Javier Heraud.

\section{RESULTADOS}

En la tabla 01 se muestra que del $100 \%$ (143) de personas adultas mayores que participaron en el estudio, el 39,2\% (56) tienen un estilo de vida saludable y un $60,8 \%$ (87) no saludable.

En la tabla 02 se muestra la distribución porcentual del estado nutricional de las personas adultas mayores que residen en la H. U. P. "Javier Heraud" del distrito de Santa, prevaleciendo el estado nutricional normal con un 42,0\% (60), seguido por el $27,2 \%$ (39) de personas con sobrepeso, 18,2\% (26) delgadez y $12,6 \%$ (18) obesidad. 
Tabla 01

ESTILO DE VIDA DEL ADULTO MAYOR EN LA

H.U.P. JAVIER HERAUD - SANTA, 2011

\begin{tabular}{lcc}
\hline Estilo de vida & Frecuencia & Porcentaje \\
\hline Saludable & 56 & 39,2 \\
No saludable & $\mathbf{8 7}$ & $\mathbf{6 0 , 8}$ \\
\hline Total & 143 & 100,0 \\
\hline
\end{tabular}

Fuente: Escala de estilo de vida. Modificado Por: Díaz, R; Reyna, E; Delgado, R. Aplicado a los adultos mayores de 60 años a más de la H.U.P. Javier Heraud - Santa, Enero 2011.

Tabla 02

ESTADO NUTRICIONAL DEL ADULTO MAYOR

\begin{tabular}{lcc}
\hline Estilo nutricional & Frecuencia & Porcentaje \\
\hline Delgadez & 26 & 18,2 \\
Normal & $\mathbf{6 0}$ & $\mathbf{4 2 , 0}$ \\
Sobre peso & 39 & 27,2 \\
Obesidad & 18 & 12,6 \\
\hline Total & 143 & 100,0 \\
\hline
\end{tabular}

Fuente: Tabla de valoración del estado nutricional según índice de Masa Corporal (IMC). Adulto mayor de 60 años a más. Ministerio de Salud (MINSA) y la ficha de valoración nutricional.Silva I. Aplicado al adulto mayor del H.U.P Javier Heraud - Santa, Enero 2011.

Tabla 03

RELACIÓN ENTRE EL ESTILO DE VIDA Y EL ESTADO NUTRICIONAL DEL ADULTO MAYOR

\begin{tabular}{|c|c|c|c|c|c|c|c|c|c|c|}
\hline \multirow{3}{*}{ Estilo de vida } & \multicolumn{8}{|c|}{ Estado nutricional } & \multirow{2}{*}{\multicolumn{2}{|c|}{ Total }} \\
\hline & \multicolumn{2}{|c|}{ Delgadez } & \multicolumn{2}{|c|}{ Normal } & \multicolumn{2}{|c|}{ Sobrepeso } & \multicolumn{2}{|c|}{ Obesidad } & & \\
\hline & $\mathbf{n}$ & $\%$ & $\mathbf{n}$ & $\%$ & n & $\%$ & $\mathbf{n}$ & $\%$ & $\mathbf{n}$ & $\%$ \\
\hline Saludable & 9 & 6,3 & 29 & 20,3 & 12 & 8,4 & 6 & 4,2 & 56 & 39,2 \\
\hline No saludable & 17 & 11,9 & 31 & 21,7 & 27 & 18,9 & 12 & 8,4 & 87 & 60,8 \\
\hline Total & 26 & 18,2 & 60 & 42,0 & 39 & 27,3 & 18 & 12,6 & 143 & 100,0 \\
\hline
\end{tabular}

Fuente: Escala de estilo de vida. Modificado Por: Díaz, R; Reyna, E; Delgado, R. Tabla de valoración del estado nutricional según índice de Masa Corporal (IMC). Adulto mayor de 60 años a más. Ministerio de Salud (MINSA) y Ficha de valoración nutricional. Silva I. Aplicado al adultos mayor de la H.U.P. Javier Heraud- Santa, Enero del 2011.

Chi Cuadrado $=3,754,3 \mathrm{gl} \mathrm{p}=0,289>0,05$. 


\section{DISCUSIÓN}

Los resultados obtenidos en la H.U.P. Javier Heraud, en la Tabla 01 se muestra que la mayoría de adultos mayores presentan un estilo de vida no saludable. Posiblemente se debe a diversos factores, tales como el grado de instrucción primaria o analfabetismo, un nivel económico regular que no llega a satisfacer las necesidades básicas, adoptando conductas inadecuadas, como la falta de actividad física (sedentarismo) y una inadecuada alimentación.

Los hallazgos encontrados se relacionan con los encontrados por Elcorrobarrutia, J. y Flores, LL. ${ }^{22}$, en su estudio titulado "Estilo de vida y estado nutricional en el adulto mayor en la Urb. La Libertad" Chimbote, con una muestra de 239 adultos mayores, donde se encontró como resultado que el 78,2\% presentaron un estilo de vida no saludable y el $21,8 \%$ estilo de vida saludable.

El estilo de vida representa el conjunto de decisiones que toma el individuo sobre su salud y sobre las cuales ejerce cierto grado de control. Desde este punto de vista, las malas decisiones y los hábitos perjudiciales conllevan riesgos que se originan en el propio individuo, en el adulto mayor. Según este concepto, la calidad de vida resulta dependiente no sólo del estado biológico sino de las condiciones del medio ambiente relacionadas en un sentido amplio (Interacción social, hábitos, adecuado control y tratamiento de las tensiones y emociones negativas), un buen régimen de ejercicios, sueño y distracción; el control y la evitación del abuso de sustancias como la cafeína, nicotina y alcohol; una correcta distribución y aprovechamiento del tiempo, de manera consistente y mantenida en su vida cotidiana, y que puede ser pertinente para el mantenimiento óptimo de su salud. ${ }^{23}$

Los adultos mayores evidencian en la Tabla 02 un estado nutricional normal en un 42,0\%, aunque tienen un estilo de vida no saludable. Esto se debe a la responsabilidad y/o iniciativa de la familia en el cuidado de su salud, buscando información concerniente a una buena, adecuada, equilibrada, variada y gastronómica nutrición, siendo su comida fácil de preparar, estimulante de apetito y de fácil masticación y digestión. Además, cuentan con el apoyo y afecto familiar. Su entorno en el que viven es seguro, los integrantes de la familia se encuentran concientizados de cómo vivir saludablemente favoreciendo de esta manera su estado nutricional.

Sin embargo, los resultados obtenidos de la investigación del H.U.P Javier Heraud, muestran también cifras elevadas de sobrepeso y obesidad, teniendo un estilo de vida saludable. Esto se debe al déficit de conocimiento sobre una ade- 
cuada y balanceada alimentación de acuerdo a su etapa de vida y a factores fisiológicos de las personas adultas; presentan un desequilibrio nutricional, debido a una (alimentación inadecuada, inactividad, preocupación, estrés, sedentarismo, la soledad, abandono y a su misma pobreza).

Estos problemas de desnutrición, sobrepeso u obesidad en los adultos mayores son muy comunes. La obesidad se debe a un desbalance energético originado fundamentalmente por una ingesta calórica superior a la requerida, lo que trae como consecuencia un exceso de grasa en el organismo, que puede ser perjudicial para la salud; sin embargo, el nivel de exceso, la distribución corporal de la grasa y las consecuencias asociadas a la salud varían en forma considerable entre individuos obesos. ${ }^{24}$

$\mathrm{Al}$ relacionar la variable estilo de vida con el estado nutricional (Tabla 3 ) utilizando el estadístico Chi-cuadrado de independencia $\left(\chi^{2}=3,754,3 \mathrm{gl}\right.$ y $p>0,05)$, encontramos que no existe relación estadísticamente significativa entre ambas variables.

Los resultados encontrados muestran que hay adultos mayores que aunque tienen un estilo de vida no saludable presentan un estado nutricional normal y que presentando un estilo de vida saludable presentan elevados porcentajes de sobrepeso, delgadez y obesidad. Es probable que el estado nutricional del adulto mayor se vea influenciado por muchos factores y no solo al consumo de alimentos; por tal razón, los resultados llevan a concluir en esta investigación que el estilo de vida no tiene relación con el estado nutricional del adulto mayor.

\section{CONCLUSIONES}

1. Más de la mitad de los adultos mayores de la H.U.P "Javier Heraud" del distrito de Santa, presentan un estilo de vida no saludable y un menor porcentaje, un estilo de vida saludable.

2. En relación al estado nutricional de los adultos mayores tenemos que menos de la mitad presentan un estado nutricional normal; más de la cuarta parte, sobrepeso; menos de la quinta parte, delgadez y más de la décima parte, obesidad.

3. No existe relación estadísticamente significativa entre el estilo de vida y el estado nutricional en el adulto mayor del H. U. P. "Javier Heraud" del distrito de Santa. 


\section{REFERENCIAS BIBLIOGRÁFICAS}

1 HENDERSON A. Estilo de vida saludable. Puntos de vista para una opción actual y necesaria. [articulo en internet] Cuba 2007. [Citado 2010 Febrero 3] [Alrededor de 2 pantallas] Disponible desde el URL:http://www.psicologiacientifica.com/bv/psicologia-307-1-estilo-devida-saludable-puntos-de-vista-para-una-opcion-act.html.

2 Coronado L. Nutrición y Deporte = Formación Física. [artículo en internet]. 2006 Cuba. Departamento de Ciencias Aplicadas. Facultad de Cultura Física Universidad de Ciego de Ávila - UNICA [citado 2011 Dic 9] [Alrededor de 5 pantallas] Disponible desde: URL http://ntic.uson.mx/wikisalud/index.php/H11.

3 Conicyt Repositorio Institucional. Ficha de Iniciativa De Ciencia, Tecnología e Innovación (CIT)). Diseño y Evaluación de Alimentos Saludables Destinados al Adulto Mayor.[monografía en internet]. EEUU Noveno Concurso Nacional de Proyectos. 2001. [citado 2009 Dic 09]. [alrededor de 03 pantallas]. Disponible desde URL: http://ri.conicyt.cl/575/fo-article-11248.pdf.

4 Ministerio De SAlud. Población Adulta Mayor. 01 de Octubre Día Mundial del Adulto Mayor "Todos Envejecemos Promovamos Juntos Adultos Mayores Activos y Saludables"[monografía en internet]. Lima - Perú. 2008. [citado 2009 Nov 02]. [alrededor de 02 pantallas]. Disponible desde URL: http://www.minsa.gob.pe/portada/Especiales/2008/ adulto_mayor/principal.htm.

5 Centro de Apoyo Armonización de Salud y CulturaSplendor. Proyecto Slendor Casa Hogar para la Tercera Edad.[monografía en internet]. Lima - Perú. 2004. [citado 2009 Nov 20]. [alrededor de 04 pantallas]. Disponible desde URL: http://imagenes. tupatrocinio.com/img-bdd/documentos/Proyectoa \%20Resumido \% 20TUPATRO CINIO.doc.

6 GonzÁlez L, RodríGuez A. Planteamiento de un Centro de día Gerontológico Basado en las Condiciones Socioeconómicas, Culturales y de Salud de las Personas Mayores de sesenta años de la Ciudad de Tunja. [tesis para optar el título de Licenciada en Enfermería]. Colombia: Universidad Pedagógica de Colombia; 2004; [citada 2009 Nov 13]. [alrededor de 152 pantallas]. Disponible en URL: http://www.index-f.com/lascasas/documentos/lc0125.pdf.

7 ARANCIBIA R, HERnÁNDEZ D. Estado Nutricional y Actividad Física en el Adulto Mayor en una Población de Referencia de la Provincia Ciego de Ávila, cuba: Universidad Máximo Gómez, Cuba 2008.

8 Cid P, Merino J, Stiepovich J. Factores Biológicos y Psicosociales Predictores del Estilo de Vida Promotor de Salud. [revista médica]. Chile. Dic 2006. Vol. 134 no. 12 p 1499 ISS N 0034 - 9887.

9 Letelier L. 55\% de los Adultos Mayores tiene Problemas de Sobrepeso y Obesidad.[artículoeninternet].Chile. Consorcio Periodístico S.A. 2003. [citado 2009 Nov 08]. [alrededor de 02 pantallas]. Disponible desde el URL: http://www.icarito.cl/medio/ articulo/0,0,3255_5666_41203279,00.html.

10 Centro de Salud de Santa. Censo 2010. Población total de habitantes. Reseña Histórica del H.U.P "Javier Heraud”. [Citado 2010 Oct 15] Santa - Perú.

11 ICARITO. Adultez y Estabilidad Corporal.[monografía en internet]. México. 2006. [citado 2009 Oct 30].[alrededor de 02 pantallas]. Disponible en URL:http://biblioteca.idict. villaclara.cu/UserFiles/File/adulto\%20medio/14.Word.doc.

12 VARgas F. Programa de Educación para la Salud. Los estilos de vida de un grupo social comprenden una serie de pautas de conducta determinadas socialmente y de interpretaciones 
de situaciones sociales. [Power point en internet]. Colegio oficial de médicos Santa Cruz de Tenerife. [citado 2009 Oct 20].[alrededor de 34 pantallas]. Disponible desde el URL: http://www.comtf.es/cursos/EPS/EPS\%202004\%20Presentacion.ppt.

13 SÁnchez E. Estilos de Vida Saludables sin Tabaco.[artículo de internet]. Argentina. Publicidad por bligoo.com. 2008 [citado 2009 Oct 26]. [alrededor de 02 pantallas]. Disponible desde URL: http://elisa.bligoo.com/content/view/174244/Estilo-de-vida-saludable.html.

Vives A. Vives A. Estilo de Vida Saludable. [resumen en internet]. La Habana Cuba. 2007. [citado 2009 Nov 19].[alrededor de 09 pantallas]. Disponible desde URL: http://www.psicologiacientifica.com/bv/imprimir-307-estilo-de-vida-saludable-puntosde-vista-para-una-opcion-actual-y-necesaria.html.

WIKIPEDIA. Alimentación Humana. Enciclopedia libre.[citado 2009 Dic 05]. [alrededor de 03 pantallas]. Disponible desde URL: http://es. wikipedia.org/wiki/Alimentaci\%C3\%B3n.

INSTITUTO NACIONAL DE SALUD. El Ejercicio y su Salud. Su guía personal para mantenerse sano y fuerte. [articulo de Internet]. Estados Unidos. 2007. [citado 2009 Nov 28]. [alrededor de 06 pantallas]. Disponible desde el URL. http://www.nia.nih.gov/Espanol/ Publicaciones/Ejercicio/exercise_appendixD.htm.

17 ZALDÍVAR D. Estrés y Factores Psicosociales en la Tercera Edad. [ponencia presentada en el congreso de Longevidad. Hotel Nacional]. La Habana. 2004. [citado 2009 Oct. 21]. [alrededor de 02 pantallas]. Disponible en URL: http://www.sld.cu/saludvida/ adultomayor/temas.php?idv $=5940$.

18 LOPATEGUi E. Salud como Responsabilidad Individual.(artículo en internet). Copyright ${ }^{\odot}$ 2000. [citado 2009 Nov 30].[alrededor de 01 pantalla]. Disponible desde URL: http://www.saludmed.com/Salud/CptSalud/CptSaRes.html.

19 Mella R, González L, D' Appolonio J, Maldonado i, Fuenzalida A y díaz A. Factores Asociados al Bienestar Subjetivo en el Adulto Mayor. [serie en internet]. Santiago; 2004. Psykhe 13 [Citado 2008 Abril 4]. [Alrededor de 10 pantallas]. Disponible desde: http://www.scielo.cl/scielo.php?pid=S071822282004000100007\&script $=$ sci_arttext .

20 Ameiwaece. Autorrealización. Enciclopedia on line. [citado 2009 Dic 19]. [alrededor de 01 pantalla]. Disponible desde URL: http://www.waece.org/enciclopedia/diccionario.php.

21 Gómez J.; JuRAdo M.; Viana B.; DA M.; HernándeZ A.; Estilos y Calidad de Vida. [revista digital en internet]. Buenos Aires. 2005. [citado 2009 Nov 28]. [alrededor de 10 pantallas]. Disponible desde URL: http://www.efdeportes.com/efd90/estilos.htm.

22 Elcorrobarrutia J, Flores LL. "Estilo de Vida y Estado Nutricional en el Adulto Mayor en la Urb. La Libertad" [Tesis para obtener el título de licenciada en enfermería] Chimbote: Universidad católica los angeles de Chimbote 2009, [citado 2011 Mayo8].

Duncan P. Estilos de Vida. En Medicina en Salud Pública en el año 1986.Universidad de Caldas. Manizales 1987. [citado 2011 Mayo8]. http://www.psicomundo.com/tiempo/ cvepe2/biblio3.ht.

24 Bertran M, Pérez L, Roselló M. 2006. Postura del Colegio Mexicano de Nutriólogos sobre los problemas prioritarios de alimentación y nutrición en el país y para los programas para atenderlos. 1a ed. México, DF: Colegio Mexicano de Nutriólogos. Disponibleen:http://www.didac.ehu.es/antropo/16/16-6/BernalOrozco.pdf. 